\title{
Sistema de estimación, certificación y aprobación de reservas de hidrocarburos en México; análisis de desempeño
}

\section{The Evaluation, Certification and Approbation of Oil and Gas Reserves in Mexico; Facts and Performance}

\author{
Rodríguez-Padilla Víctor \\ Departamento de Sistemas Energéticos \\ Facultad de Ingeniería \\ Universidad Nacional Autónoma de México \\ Correo:energia123@hotmail.com
}

Información del artículo: recibido: agosto de 2012, aceptado: septiembre de 2012

\section{Resumen}

El sistema de evaluación, certificación y aprobación de reservas aplicado en México desde 2010 busca reproducir las mejores prácticas de la industria petrolera internacional. Ha ganado en rigor, sencillez, veracidad y transparencia, sin embargo, su desempeño ha sido desigual, pues ha logrado brindar mayor certeza sobre las reservas probadas, pero no ha podido disipar la incertidumbre sobre las reservas probables y posibles.

\footnotetext{
Abstract

The evaluation, certification, approval and disclosure system of reserves applied in Mexico since 2010 aims to replicate the best practices of the international oil industry. It has gained rigor, simplicity, truthfulness and transparency, however its performance has been unequal: it has achieved to provide more certitude over the proved reserves but it has failed to dissipate the uncertainty over the probable and possible reserves.
}

\section{Descriptores:}

- petróleo

- gas natural

- reservas

- evaluación

- divulgación

\section{Keywords:}

- oil

- natural gas

- reserves

- evaluation

- disclosure 


\section{Introducción}

El petróleo es la fuente de energía comercial más utilizada en el mundo y lo seguirá siendo en las próximas décadas. Resulta entonces fundamental conocer su disponibilidad para prever su remplazo sin angustias ni precipitaciones. Para el ingeniero petrolero es fundamental saber con la mayor exactitud posible las reservas de un campo para establecer el plan de desarrollo que maximice la recuperación de los hidrocarburos in situ. Para las empresas petroleras las reservas son materia de trabajo, activo a desarrollar y monetizar. La compra de derechos de explotación, la participación en proyectos y los financiamientos se deciden en función de la cantidad de petróleo o gas que puede ser registrada contablemente, el volumen que puede producirse y el retorno de la inversión. Para la industria petrolera las reservas son el corazón de la confianza y la credibilidad que aseguran acceso a fondos para desarrollar proyectos e innovaciones que respondan al crecimiento de la demanda. Para el sector financiero las reservas son una medida del valor de una empresa petrolera y por lo tanto fundamento de su capacidad crediticia. Para los países exportadores altamente dependientes de los ingresos petroleros, el caso de México, ${ }^{1}$ es particularmente importante saber por cuánto tiempo más podrán seguir utilizando ese recurso natural no renovable como palanca de desarrollo.

Cuál es la dotación original en hidrocarburos, cuánto se ha descubierto, cuánto se ha recuperado, cuánto se espera recuperar en el futuro y cuál es su valor de mercado, es información estratégica que ingenieros, empresarios, poderes públicos y financistas necesitan conocer con la mayor exactitud posible. La calidad de dicha información dependerá del buen diseño y funcionamiento de los sistemas de evaluación, certificación, aprobación y publicación de reservas.

La literatura ha estudiado el tema de las reservas desde varios puntos de vista. Algunos investigadores ponen el acento en el ciclo de vida, en sus fases de ascenso, clímax y declinación. Otros focalizan sobre la concentración geográfica y la geopolítica. Los que fijan la atención en los aspectos técnicos son numerosos. El valor económico de las reservas es una de las áreas donde la literatura es también abundante. La importancia de las reservas para la firma, así como el proceso de certificación y notificación a las autoridades reguladoras por parte de las firmas petroleras y de ingeniería son dos temas tratados regularmente por los especialistas.

1 En México entre el 30 y 40\% de los ingresos de la federación dependen del petróleo.
El objeto de este artículo es evaluar la capacidad del sistema de estimación, certificación y aprobación de reservas aplicado en México en su capacidad para reducir la incertidumbre para los actores que utilizan esta variable clave para la toma de decisiones.

El artículo se divide en dos partes: la primera aborda las dificultades conceptuales y prácticas que enfrenta el explorador cuando cataloga hidrocarburos como reservas, así como el sistema utilizado en México desde 2010. En la segunda parte se analiza el desempeño de dicho sistema y se discuten posibles mejoras.

\section{Desarrollo}

\section{Dificultades conceptuales y prácticas}

Aunque se trata de un indicador clave en la industria petrolera internacional el concepto de reservas es objeto de debates académicos sobre su significado y alcance, pues su connotación es a la vez económica y geológica y su cálculo ocurre en un contexto de incertidumbre e interpretación subjetiva. Las controversias giran también en torno a las características de los hidrocarburos, a la confiabilidad de la estimación, tanto en volumen como en valor, así como al rigor y profesionalismo en el proceso de certificación y a la transparencia en la divulgación de las cifras.

La dificultad para tratar un tema complejo por naturaleza aumenta por la ambigüedad con la que se utiliza el término "reservas", por un lado, para referirse de manera genérica a las estimaciones que unidades especializadas de las empresas petroleras reportan a sus directivos con fines de planeación y gestión del portafolio de proyectos, por otro lado, para denominar a las cantidades de hidrocarburos que compañías especializadas certifican a partir de criterios regulatorios, los volúmenes que son reportados ante las autoridades, los que dan a conocer los gobiernos y los que aparecen en las revistas especializadas y los anuarios de organismos internacionales. El explorador, el economista, el financiero, los poderes públicos hablan de reservas, pero rara vez comparten el mismo significado.

A los problemas de claridad y precisión se agregan los de veracidad, transparencia y credibilidad. Lo que está en juego produce un fuerte incentivo en algunos actores para cubrir las reservas con un velo de opacidad, exagerando o temperando las cifras en función de las circunstancias.

Algunas compañías minimizan los volúmenes que yacen en el subsuelo para solicitar incentivos fiscales y ventajas en los contratos de exploración y producción. Otras, sobre estiman su importancia para mejorar el 
atractivo de las acciones de la empresa en el mercado de valores o para conseguir más y mejores créditos en los mercados de capital.

Algunos gobiernos son propensos a tomar ventaja de la asimetría de información por razones de conveniencia económica, política, diplomática o estratégica. Algunos consideran el tema como sensible y las cifras como secreto de Estado. Sobrevalorar las reservas es una medida utilizada para reivindicar cuotas de producción más importantes en el seno de la Organización de Países Exportadores de Petróleo (OPEP), atraer inversión, detonar alianzas, ampliar espacios en foros internacionales, obtener reconocimiento y prestigio (Salameh, 2004).

La incertidumbre sobre las reservas podría disminuir sustancialmente con una regulación internacional, sin embargo, en la práctica poco se ha avanzado al no tener los grandes países exportadores interés en ello. En países con instituciones fuertes -los altamente desarrollados- las autoridades encargadas del correcto funcionamiento de los mercados de valores han establecido regulaciones que las compañías petroleras deben seguir escrupulosamente para estimar el valor de sus activos en tierra; esa normatividad también se aplican a las compañías públicas de países productores que se fondean en dichos mercados, por lo que dicha normatividad adquiere un carácter supranacional.

A pesar del gran avance tecnológico sigue siendo incierto del volumen de petróleo y gas in situ y el que eventualmente podría recuperarse, particularmente en el caso de las reservas probables y posibles (Etherington, 2004). Es fácil saber con gran exactitud las toneladas de acero, cemento o vidrio que se producen cada año, pues las fábricas y la producción están a la vista y se puede medir. En cambio, los yacimientos están bajo tierra y no es fácil conocer sus dimensiones exactas ni su contenido. Los volúmenes de líquido y gas no pueden inspeccionarse físicamente o enumerase exactamente, pues no son inventarios. En esas condiciones sólo es posible hacer una estimación mediante métodos determinísticos, probabilísticos y mixtos (Patricelli y McMichael, 1995).

Las reservas no son la cantidad de petróleo que contiene un yacimiento, sino la cantidad que puede llegar a extraerse con la tecnología y en las condiciones económicas prevalecientes en el momento de la evaluación. Sabemos que son finitas, de eso no hay duda, sin embargo nadie sabe qué tan finitas son. Además dependen del precio del petróleo (Farzin, 2001). Algunos investigadores consideran que la tecnología y la economía pesan más que la geología en la evolución de las reservas (Maugeri, 2004).
El valor de un activo petrolero es función de su capacidad efectiva o potencial para generar una utilidad en el futuro. Las reservas son activos, pero su valuación requiere conocer o estimar el volumen del petróleo in situ, las cantidades recuperables, los volúmenes que serán efectivamente vendidos, los costos de producción y los ingresos que serán generados (Etherington, 2005). Sobre todas esas variables pesa una gran incertidumbre.

Aunque se aplique la tecnología más moderna sólo una pequeña fracción del yacimiento se conoce físicamente, por lo que las mediciones de sus propiedades son por lo general indirectas. La manera como un yacimiento responderá a un programa específico de desarrollo es igualmente incierta. La estimación de las cantidades recuperables y comercializables de un yacimiento es en última instancia una estimación de un rango de perfiles potenciales de producción y de su integración temporal. La evaluación de los componentes de valor es incierta, pues se requiere estimar costos, precios e impuestos a lo largo del ciclo de vida del proyecto.

La estimación de reservas tiene elementos subjetivos, especialmente cuando se evalúa el rango de incertidumbre técnica, de ahí que diferentes certificadores pueden generar diferentes estimaciones (Worthington, 2005). La situación se ve agravada por la ausencia de protocolos aceptados universalmente para abordar algunas de las cuestiones de interpretación más polémicas en ciencias de la tierra y geoingeniería. A menudo, los usuarios de la información de reservas suponen que las metodologías técnicas que sustentan las cifras reportadas son muy sólidas, pero no es así.

Hodgin y Harrell (2007) sintetizan la problemática señalando que la estimación de reservas es un proceso complejo que implica la integración de diversas disciplinas técnicas y se basa en una combinación de conocimientos, experiencia y juicio. Las reflexiones de los evaluadores se ven influidas por la interpretación de datos técnicos y el conocimiento de las definiciones aplicables.

La terminología utilizada en la clasificación de recursos y reservas ha sido objeto de estudio y revisiones internacionales desde mediados de 1930. Durante ocho décadas organizaciones técnicas, autoridades reguladoras e instituciones financieras han introducido una terminología cada vez más compleja. De manera paralela la evolución de la tecnología ha producido métodos de ingeniería cada vez más precisos para evaluar reservas y ha intensificado la necesidad de mejorar la uniformidad y coherencia del lenguaje entre los profesionales del ramo. 
La Society of Petroleum Engineers (SPE), el World Petroleum Council (WPC), la American Association of Petroleum Geologists (AAPG) y la Society of Petroleum Evaluation Engineers (SPEE) se han puesto de acuerdo en el Petroleum Resources Management System (PRMS) ${ }^{2}$, que en los últimos años proporciona un estándar internacional de clasificación y notificación de reservas y recursos. Dicho sistema ha sido adoptado directamente o en versiones modificadas por muchas empresas petroleras como parte de sus sistemas internos de gestión de activos (Harrel, 2008). La Society of Exploration Geophysicists (SEG) se ha unido al grupo y las cinco asociaciones han publicado una guía de aplicación ${ }^{3}$.

Los reguladores financieros no han logrado la convergencia alcanzada por las organizaciones técnicas. Las regulaciones financieras en materia de reservas contemplan que las empresas de la industria del petróleo y el gas notifiquen de los volúmenes de reserva en el subsuelo y una proyección razonable de los flujos de efectivo asociados con su desarrollo, sin embargo no hay un sistema universal. La agencia que ha tenido la mayor influencia en el establecimiento de normas ha sido la Comisión de Valores de los Estados Unidos (Security and Exchange Comisión -SEC-); las normas que aplica desde 2010 están alineadas con los principios y directrices de evaluación de reserva contenida en el PRMS, aunque con algunas diferencias ${ }^{4}$.

2 SPE, AAPG, WPC y SPEE (2007).

3 Véase: SPE, AAPG, WPC, SPEE y SEG (2011).

4 Esa alineación ahora permite a las empresas gestionar sus negocios con base en los estándares del PRMS, pero adaptarse a las regulaciones de la SEC dentro de un sistema de evaluación integrado (Virine y MacDonald, 2009). Para una comparación entre las reglas de la SEC y el PRMS véase Etherington (2009). Las nuevas regulaciones permiten, entre otras cosas: calcular el valor de las reservas utilizando el precio promedio anual en lugar del precio de fin de año; incluir recursos no tradicionales como las arenas asfálticas, los esquistos bituminosos y el gas de yacimientos de carbón; asimismo, notificar las reservas probables y posibles; realizar la evaluación utilizando el criterio de "certeza razonable" en lugar del criterio de "certitud". Las nuevas reglas ya no requieren que un número limitado de tecnologías, rígidas y específicas se utilice para establecer el nivel de certidumbre de las reservas que revela una compañía. Olsen, Lee y Blasingame (2010) califican ese nuevo derrotero de "liberalización técnica" y advierten del peligro de sobre estimación de reservas. Lee (2010) analiza el concepto de "tecnología confiable", pieza clave de los nuevos criterios utilizados para clasificar los recursos como reservas probadas, probables o posibles. Sobre la manera como los recursos no convencionales deben ser tomados en cuenta bajo la nueva regulación de la SEC véase Lee (2009b).
El sistema bajo análisis

Desde 2003 Petróleos Mexicanos, a través del organismo subsidiario Pemex Exploración y Producción (PEP) estima las reservas probadas siguiendo los criterios y lineamientos de la SEC, incluyendo los que entraron en vigor el 1 de enero de $2010^{5}$. Para las reservas probables y posibles emplean criterios y lineamientos convenidos por las organizaciones técnicas internacionales citadas (SPE, AAPG, WPC y SPEE, 2007 y 2011) ${ }^{6}$. Los resultados son dados a conocer en el informe denominado: "Reservas de Hidrocarburos de México", donde se asientan las definiciones utilizadas en el proceso de estimación, certificación y aprobación (Apéndice), (Pemex, 2010 y 2011)

PEP elabora estimaciones por región petrolera (marina noreste, marina suroeste, norte y sur) y por grado de certeza (reserva probada, probable, posible, y sus agrupaciones $2 \mathrm{P}$ y $3 \mathrm{P}$ ). Las reservas probadas se desagregan en desarrolladas y no desarrolladas. La estimación se realiza al 1 de enero de cada año. Las unidades que se utilizan en la evaluación son barriles de petróleo para hidrocarburos líquidos y millones de pies cúbicos para gas natural. Para llegar a una cifra global utilizan los poderes caloríficos de cada componente y el resultado se expresa en millones de barriles de petróleo crudo equivalente. ${ }^{8}$

A partir de 2010 la estimación de reservas realizada por PEP es sometida a un proceso de la certificación, aprobación y publicación previsto en la regulación?:

- Empresas internacionales especializadas revisan que las estimaciones de PEP se hayan realizado de manera rigurosa y sus resultados sean confiables ${ }^{10}$. Las cifras estimadas por el organismo son válidas si

5 Véase: SEC (2010).

6 Véase: SPE, AAPG, WPC y SPEE (2007 y 2011)

7 Véase: Pemex (2010 y 2011)

8 Estimar las reservas en términos de petróleo crudo equivalente no es común en la industria petrolera internacional ni entre los organismos internacionales. En México se hace desde mediados de la década de los años 70 del siglo pasado y tiene la ventaja de hacer más cuantiosa las reservas.

9 El procedimiento está previsto fundamentalmente en el artículo 10 del Reglamento de la Ley Reglamentaria del Artículo 27 Constitucional en el Ramo del Petróleo.

10 Pemex contrató a las empresas Ryder Scott Company, Netherland Sewell International, Netherland Sewell International y DeGolyer and MacNaughton, para certificar las reservas en la región norte, sur, marina noreste y marina suroeste, respectivamente. 
difieren en menos de $10 \%$ de las obtenidas por los certificadores. Para evitar conflicto de intereses la paraestatal debe abstenerse de contratar para otros fines a los terceros que realicen certificaciones durante los doce meses siguientes a dicha certificación.

- La Comisión Nacional de Hidrocarburos (CNH) revisa los informes finales de las empresas certificadoras y, en su caso, les da el visto bueno. También revisa la estimación realizada por PEP y, en su caso, la aprueba; si el dictamen es negativo tiene facultades para establecer las cifras que considere adecuadas (CNH, 2010c).

- Con base en la información proporcionada por la $\mathrm{CNH}$ la Secretaría de Energía inscribe en el Registro Petrolero las reservas oficiales de hidrocarburos; con ese acto da a conocer formalmente las reservas petroleras del país (Sener, 2010).

- El Comité de Auditoría y Evaluación del Desempeño (CAED) del Consejo de Administración de Pemex emite una opinión sobre la estimación de reservas y el cumplimiento de las metas de restitución.

Se trata de un procedimiento riguroso, sencillo, ordenado y transparente, sin embargo, algunos vacíos, zonas grises y traslapes contenidos en el marco regulatorio son fuente de incertidumbre.

\section{Discusión y análisis de resultados}

El cuadro 1 presenta los resultados de la aplicación del sistema de estimación y aprobación de reservas en 2010 y 2011. Las coincidencias entre los participantes en el proceso son notables en reservas probadas, pero divergen en los otros niveles. Por ejemplo, las estimaciones de Pemex y sus certificadores coincidieron sólo en $31.3 \%$ y en $56.7 \%$ para las reservas $2 \mathrm{P}$ y $3 \mathrm{P}$ de la región norte en 2010; la brecha se profundizó al año siguiente. Las diferencias también son apreciables cuando se comparan los resultados de Pemex con los de la CNH. El origen de tales divergencias radica fundamentalmente en el petróleo y gas que contiene el activo Aceite Terciario del Golfo (ATG) más conocido como Chicontepec. ${ }^{11}$

11 De acuerdo con Lajous (2011), las diferencias entre Pemex y su auditor respecto a las estimaciones de reservas de hidrocarburos 3P de Chicontepec ascendieron en 2010 a 45\%, y la correspondiente a las $2 \mathrm{P}$ a $75 \%$. En 2011 estas mismas diferencias fueron de $58 \%$ y $75 \%$, respectivamente.

Cuadro 1. Reservas de hidrocarburos en 2010 Y 2011 (millones de barriles de petróleo crudo equivalente)

\begin{tabular}{|c|c|c|c|c|c|c|}
\hline & \multicolumn{3}{|c|}{ Reservas al 1 de enero de 2010} & \multicolumn{3}{|c|}{ Reservas al 1 de enero de 2011} \\
\hline & $1 \mathrm{P}$ & $2 \mathrm{P}$ & $3 \mathrm{P}$ & $1 \mathrm{P}$ & $2 \mathrm{P}$ & $3 \mathrm{P}$ \\
\hline \multicolumn{7}{|c|}{ Estimadas por Pemex Exploración y Producción } \\
\hline Marina Noreste & 6,712 & 9,191 & 12,097 & 6,283 & 9,368 & 12,081 \\
\hline Marina Suroeste & 1,892 & 3,421 & 6,011 & 2,076 & 3,776 & 6,384 \\
\hline Norte & 1,352 & 10,502 & 19,142 & 1,436 & 10,496 & 18,884 \\
\hline Sur & 4,036 & 5,113 & 5,824 & 4,000 & 5,169 & 5,725 \\
\hline Total & 13,992 & 28,229 & 43,075 & 13,796 & 28,809 & 43,074 \\
\hline \multicolumn{7}{|c|}{ Estimadas por Terceros Independientes } \\
\hline Marina Noreste & 6,599 & 8,603 & 12,004 & 6,578 & 8,689 & 11,353 \\
\hline Marina Suroeste & 1,727 & 3,360 & 5,996 & 1,968 & 3,928 & 6,776 \\
\hline Norte & 1,260 & 3,289 & 10,844 & 1,387 & 3,492 & 8,770 \\
\hline Sur & 3,802 & 4,891 & 5,578 & 3,682 & 4,759 & 5,610 \\
\hline Total & 13,388 & 20,143 & 34,421 & 13,616 & 20,869 & 32,510 \\
\hline \multicolumn{7}{|c|}{ Aprobadas por la Comisión Nacional de Hidrocarburos } \\
\hline Marina Noreste & 6,712 & 9,191 & 12,097 & 6,283 & 9,368 & 12,054 \\
\hline Marina Suroeste & 1,892 & 3,421 & 6,011 & 2,076 & 3,776 & 6,384 \\
\hline Norte & 1,352 & En Revisión & En Revisión & 1,436 & 3,492 & 8,770 \\
\hline Sur & 4,036 & 5,113 & 5,824 & 4,000 & 5,169 & 5,725 \\
\hline Total & 13,992 & No disponible & No disponible & 13,796 & 21,805 & 32,933 \\
\hline \multicolumn{7}{|c|}{ Publicadas por la Secretaría de Energía en el Registro Petrolero (cifras oficiales para México) } \\
\hline Marina Noreste & 6,712 & 9,191 & 12,097 & 6,283 & En Revisión & En Revisión \\
\hline Marina Suroeste & 1,892 & 3,421 & 6,011 & 2,076 & En Revisión & En Revisión \\
\hline Norte & 1,352 & En Revisión & 19,142 & 1,436 & En Revisión & En Revisión \\
\hline Sur & 4,036 & 5,113 & 5,824 & 4,000 & En Revisión & En Revisión \\
\hline Total & 13,992 & No disponible & 43,075 & 13,796 & En Revisión & En Revisión \\
\hline
\end{tabular}

Notas. 1. Cifras al 1 de enero de cada año. En la página del Registro Petrolero Sener advierte que "para 2010 las reservas probables y posibles correspondientes a la Región Norte están en proceso de revisión por parte de la CNH por lo que los valores mostrados --(3P)-- fueron proporcionados por Pemex". http://egob2.energia. gob. $\mathrm{mx} / \mathrm{SNIH} /$ Reportes $/ \mathrm{P}=$ reservas probadas; $2 \mathrm{P}=$ reservas probadas y posibles; $3 \mathrm{P}=$ reservas probadas, probables y posibles).

Fuente: elaboración del autor con cifras de la Pemex (2010 y 2011), CNH (2010 a y b, 2011a; Sener (2010). 
Los dos primeros años de aplicación del sistema permitieron relevar tres debilidades del sistema. Una primera fuente de incertidumbre se refiere a las atribuciones de los participantes en materia de difusión de cifras. Pemex publica sus resultados con independencia de las conclusiones de las empresas certificadoras, el dictamen de la CNH y las cifras que Sener inscribe en el Registro Petrolero. Tradicionalmente la paraestatal da a conocer su estimación el 18 de marzo y la plasma en reportes públicos, entre ellos a la SEC y a la Bolsa Mexicana de Valores, ${ }^{12}$ que incluyen notas de advertencia sobre el carácter preliminar de las cifras y su posterior revisión y aprobación por parte de la autoridad competente. Por su parte, la CNH da a conocer las reservas que considera válidas ya sea retomando las cifras de Pemex o las de los certificadores, pero también haciendo sus propias estimaciones. Finalmente, Sener retoma parcialmente las cifras de la $\mathrm{CNH}$ y las complementa con las de Pemex. Como resultado en un momento dado circulan varias versiones de reservas.

Una segunda fuente de incertidumbre deriva de las responsabilidades y facultades de la $\mathrm{CNH}$ en un contexto de fuertes asimetrías institucionales. El Congreso creó la $\mathrm{CNH}$ como una de las piezas clave de un nuevo esquema organizativo acorde con una industria petrolera más abierta y competitiva que remplazaría paulatinamente al monopolio público y donde estuvieran claramente diferenciadas la operación, la regulación y la política pública. Como se trataba de un primer paso los legisladores fueron prudentes y le asignaron, sobre todo, tareas de apoyo y consulta. En materia de reservas fue distinto, pues le dieron atribuciones superiores a cualquier otra autoridad con el ánimo de garantizar objetividad, credibilidad y certidumbre. Ese privilegio ha generado resistencias, quizás porque se estima que el regulador aún no tiene la solvencia técnica para la tarea encomendada, pues la experiencia y recursos técnicos, materiales y humanos con los que cuenta la $\mathrm{CNH}$ son sustancialmente menos importantes que aquellos con los que cuenta PEP. Las resistencias del operador y de la autoridad tutelar del sector se han hecho patente sobre todo cuando el regulador ha tomado decisiones autónomas.

En 2010 la CNH decidió otorgar el visto bueno a los reportes de los terceros independientes, así como apro bar los reportes de PEP con excepción de los correspondientes a las de reservas $2 \mathrm{P}$ y $3 \mathrm{P}$ de la región norte; las cifras cuestionadas quedaron sujetas a un proceso de revisión. Una consecuencia de esa decisión fue dejar en suspenso las reservas totales del país. En 2011 y sin haber resuelto el impase, la CNH otorgó el visto bueno a los informes de los certificadores, aprobó los reportes de PEP, pero nuevamente rechazó los correspondientes a las reservas $2 \mathrm{P}$ y $3 \mathrm{P}$ de la región norte. ${ }^{13}$ Para evitar ambigüedades decidió ejercer plenamente sus facultades y establecer sus propias cifras, las cuales quedaron muy por debajo de las estimaciones de Pemex aunque muy cerca de las cifras de los terceros independientes (cuadro 1). El regulador también señaló que un volumen de 12,302 MMbpce, 72\% de las reservas 3P estimadas por PEP en Chicontepec, eran recurso contingente, es decir, no clasificable como reservas. Esas resoluciones pusieron a la Sener en un predicamento. El registro y difusión de las cifras oficiales con base únicamente en la información proporcionada por la $\mathrm{CNH}$ significaría una reducción sustancial de reservas del país y eventualmente consecuencias negativas en la calidad crediticia y la credibilidad del gobierno federal y sus instituciones. Para salvar la situación Sener retrasó la publicación oficial y luego retomó parcialmente las cifras de la $\mathrm{CNH}$ señalando procesos de revisión en curso.

Un tercer problema es el escaso blindaje contra presiones institucionales. Como los reportes de los certificadores y las resoluciones de la $\mathrm{CNH}$ impidieron oficializar las estimaciones de reservas $2 \mathrm{P}$ y $3 \mathrm{P}$ de la región norte y del total nacional elaboradas por Pemex para 2011, la paraestatal se vio imposibilitada para incluir sus estimaciones de reservas probables y posibles en el informe anual que rinde a la SEC; de igual modo no le fue posible alcanzar la meta de restituir las reservas $3 \mathrm{P}$ al cien por ciento, una de las metas insignia del gobierno federal. Frente a ese doble inconveniente, Pemex proporcionó nueva información geológica al auditor de la región norte para que rectificara su dictamen. Paralelamente Sener buscó una corrección por parte de la CNH. La publicidad oficial y las declaraciones presidenciales sobre el cumplimiento de la meta de restitución de reservas 3P intensificaron las presiones sobre los certificadores y la $\mathrm{CNH} .{ }^{14}$
12 En junio de cada año Pemex debe entregar a la SEC un extenso informe anual sobre el desempeño de la empresa denominado F-20, disponible en:

http://www.ri.pemex.com/files/content/PEMEX_2010_Form_20-F_ as_filed_June_30_2011.pdf,
13 La CNH atribuyó la diferencia entre Pemex y el tercero independiente a la estimación relativa al Activo Aceite Terciario del Golfo.

14 Para una descripción detallada de las tensiones durante el proceso véase Lajous (2011). 
Para 2012 las divergencias desaparecieron y el proceso fue terso. La CNH (2012) aprobó las estimaciones de Pemex para las tres categorías de reservas y las cuatro regiones (tabla 2). Éste fue el resultado de más y mejor información geológica, las lecciones aprendidas de los procesos anteriores y la mejor comprensión de las facultades de los distintos participantes. Sin embargo, como subsisten deficiencias en la arquitectura regulatoria podrían surgir nuevas controversias.

Cuadro 2. Sener, $\mathrm{CNH}$, Pemex, reservas de hidrocarburos en 2012 (millones de barriles de petróleo crudo equivalente)

\begin{tabular}{cccc}
\hline Región & $1 \mathrm{P}$ & $2 \mathrm{P}$ & $3 \mathrm{P}$ \\
\hline Marina Noreste & $6,139.40$ & $9,343.00$ & $12,526.30$ \\
Marina Suroeste & $2,115.50$ & $4,091.90$ & $7,054.40$ \\
Norte & $1,575.20$ & $7,744.50$ & $18,689.00$ \\
Sur & $3,980.20$ & $4,983.60$ & $5,567.70$ \\
Total & $13,810.30$ & $26,163.00$ & $43,837.30$ \\
\hline
\end{tabular}

Fuente CNH (2012)

\section{Conclusiones}

A pesar del gran avance tecnológico sigue siendo incierto el volumen de petróleo y gas que yace en el subsuelo y el que eventualmente puede recuperarse, particularmente en el caso de las reservas probables y posibles. Las organizaciones profesionales de la industria petrolera han desarrollado un gran esfuerzo en las últimas dos década para contar con estándares internacionales.

Después de la reforma energética de 2008 y la creación de la CNH el sistema de evaluación, certificación, aprobación y publicación de reservas de hidrocarburos en México es más riguroso, ordenado y transparente, pero prevalecen imperfecciones; hay traslapes en las facultades y responsabilidades de los participantes y el sistema no está blindado contra presiones institucionales. Esas fallas surgieron durante los procesos de 2010 y 2011; el primero quedó inconcluso y el segundo observó tensiones. El desempeño del sistema es desigual: ha logrado brindar certeza sobre las reservas probadas, pero no ha podido disipar la incertidumbre sobre las demás categorías (probables, posibles, 2P y 3P). En ambas ocasiones, especialmente en 2011, se hicieron evidentes fallas en la arquitectura regulatoria, así como en la resistencia cuando las estimaciones y decisiones del regulador difieren de las del resto de las instituciones sectoriales.

El nuevo sistema podría mejorar notablemente, en primer lugar, si CNH y Sener distinguen por separado reservas de aceite, gas natural y petróleo crudo equivalente, así como reservas probadas (desarrolladas y no desarrolladas), probables y posibles. Hasta ahora han trabajado con reservas $1 \mathrm{P}, 2 \mathrm{P}, 3 \mathrm{P}$ en petróleo crudo equivalente, pero resulta insuficiente frente al trabajo de los certificadores y los compromisos internacionales de Pemex ante la SEC. En segundo lugar, si la certificación se obtiene únicamente cuando la brecha entre la paraestatal y el certificador y, viceversa, entre el certificador y la paraestatal, es menor que $10 \%$ en ambos casos, tanto en gas como en aceite. Por su parte, Pemex debe analizar separadamente la tasa de restitución de aceite y gas, pues cuando se analizan cifras agregadas en petróleo crudo equivalente, como se hace actualmente, se esconde un menor desempeño en renovar las reservas de aceite; dicha separación sería congruente con los criterios de la SEC.

El nuevo sistema ganaría credibilidad si la $\mathrm{CNH}$ contrata a los certificadores. Para preservar la imparcialidad se debe respetar el criterio de no contratar al mismo auditor por más de cuatro años consecutivos respecto a la misma región. Cuando los volúmenes no estén certificados no deberían considerarse reservas. Aunque Pemex ya tiene la posibilidad de incluir su estimación de reservas $2 \mathrm{P}$ y $3 \mathrm{P}$ en su reporte a la SEC no debería hacerlo hasta que las cifras no hubieran sido depuradas, ordenadas y certificadas. Una vez que la $\mathrm{CNH}$ y Sener hayan establecido y publicado, respectivamente las cifras oficiales, Pemex debería considerarlas como suyas e incluirlas en sus informes, anuarios, reportes y demás publicaciones.

\section{Apendice}

Definiciones relevantes del sistema de evaluación, certificación y aprobación de reservas de hidrocarburos en México (Pemex 2010 y 2011)

Volumen original de hidrocarburos total in situ. Es la cuantificación referida a condiciones de yacimiento de todas las acumulaciones de hidrocarburos naturales. Incluye a las acumulaciones descubiertas, las cuales pueden ser comerciales o no, recuperables o no, a la producción obtenida de los campos explotados o en explotación, así como también a los volúmenes estimados en los yacimientos que podrían ser descubiertos.

Recursos prospectivos. Es la porción potencialmente recuperable del volumen original de hidrocarburos no descubierto. En otras palabras, es el volumen de hidrocarburos estimado, a una cierta fecha, de acumulaciones que todavía no se descubren, pero que han sido inferidas y que se estiman potencialmente recuperables, mediante la aplicación de proyectos de desarrollo futuros. 
Recursos contingentes. Son aquellas cantidades de hidrocarburos que son estimadas, a una fecha dada, para ser potencialmente recuperables de acumulaciones conocidas, pero el proyecto para su explotación comercial aún no se considera suficientemente maduro, por no existir mercados viables, o porque la recuperación comercial depende de tecnologías en desarrollo, o la evaluación de la acumulación es insuficiente para evaluar claramente su comercialidad.

Reservas originales (recurso económico). Son las reservas originales probadas (producción acumulada más reservas probadas) y no probadas (reservas probables y posibles). En otras palabras, es la suma de la producción acumulada y las reservas remanentes (reservas $3 \mathrm{P}$ ).

Reservas. Son las cantidades de hidrocarburos que se prevé serán recuperadas comercialmente, mediante la aplicación de proyectos de desarrollo, de acumulaciones conocidas, desde una cierta fecha en adelante, bajo condiciones definidas. Las reservas deben satisfacer cuatro criterios adicionales: i) ya estar descubiertas, ii) ser recuperables, iii) ser comerciales y iv) mantenerse sustentadas a la fecha de evaluación en uno o varios proyectos de desarrollo. Los volúmenes que siendo susceptibles de considerarse reservas, pero que no satisfagan los requerimientos de comercialización deben clasificarse como recursos contingentes. Cuando se habla de reservas se entiende la suma de reservas probadas, probables y posibles, suma también conocida como reservas $3 \mathrm{P}$.

Reservas probadas. De acuerdo con la SEC, son las cantidades estimadas de petróleo crudo, gas natural y líquidos del gas natural, para las cuales se puede demostrar con certidumbre razonable, mediante datos de geociencias y de ingeniería, que serán recuperadas comercialmente en años futuros de yacimientos conocidos bajo condiciones económicas, métodos de operación y regulaciones gubernamentales existentes a una fecha específica. Se clasifican en desarrolladas o no desarrolladas.

Reservas probadas desarrolladas. Son las reservas que se podrán recuperar con los pozos y la infraestructura existentes

Reservas probadas no desarrolladas. Son reservas que se espera recuperar a través de pozos nuevos en áreas no perforadas, o donde se requiere una inversión relativamente grande para terminar los pozos existentes y/o construir las instalaciones para iniciar la producción y transporte.
Reservas probables. Son aquellas reservas no probadas para las cuales el análisis de la información geológica y de ingeniería del yacimiento sugiere que son más factibles de no ser comercialmente recuperables, que de lo contrario. Si se emplean métodos probabilistas para su evaluación, existirá una probabilidad de al menos $50 \%$ de que las cantidades a recuperar sean iguales o mayores que la suma de las reservas probadas más probables.

Reservas posibles. Son los volúmenes de hidrocarburos cuya información geológica y de ingeniería sugiere que es menos factible su recuperación comercial que las reservas probables. De acuerdo con esta definición, cuando son utilizados métodos probabilistas, la suma de las reservas probadas más probables más posibles tendrá al menos una probabilidad de $10 \%$ de que las cantidades realmente recuperadas sean iguales o mayores.

Reservas 1P. Son las reservas probadas.

Reservas $2 P$. Son las reservas probadas y probables.

Reservas 3P. Son la suma de reservas probadas, pro bables y posibles.

\section{Referencias}

Comisión Nacional de Hidrocarburos. Resolución CNH.06.001/10, 30 de junio, 2010a [en línea]. Disponible en: http://www.cnh. gob.mx/_docs/Resoluciones/RESOLUCION_CNH2010_ RESERVAS_30_062010quintus\%20CNH_06_001_10.pdf

Comisión Nacional de Hidrocarburos. Resolución CNH.E.04. 001/10, 30 de septiembre, 2010b [en línea]. Disponible en: http://www.cnh.gob.mx/_docs/Resoluciones/RESOLUCION_ CNH2010_RESERVAS_2P_y_3P\%20CNH_E_04_001_10.pdf

Comisión Nacional de Hidrocarburos. Lineamientos que regulan el procedimiento de dictaminación para la aprobación de los reportes de evaluación o cuantificación de las reservas de hidrocarburos elaborados por Petróleos Mexicanos y el visto bueno a los reportes finales de las certificaciones realizadas por terceros independientes. Diario Oficial de la Federación, martes 14 de diciembre, 2010c [en línea]. Disponible en: http:// www.cnh.gob.mx/_docs/Resoluciones/lineamiento_reservas_ unificado_dof.pdf

Comisión Nacional de Hidrocarburos. Resolución CNH.E.03.001/ 11 relativa a la evaluación, cuantificación, y verificación de las reservas de petróleo, así como a los reportes de evaluación o cuantificación de las reservas de hidrocarburos elaborados por Petróleos Mexicanos y los reportes finales de las certificaciones de las mismas realizadas por terceros independientes, 
2011a [en línea]. Disponible en: http://www.cnh.gob.mx/_ docs/resoluciones/RESOLUCION_CNH2011_RESERVAS.pdf

Comisión Nacional de Hidrocarburos. Boletín de prensa 6 de marzo, 2012 [en línea]. Disponible en: http://www.cnh.gob.mx/_ docs/Boletin_Reservas_2012.pdf

Etherington J.R. y Phillip C. Competitive Impact of New Canadian Reserve Guidelines, en: SPE Hydrocarbon Economics and Evaluation Symposium, 5-8 de abril, 2003, Dallas, Texas.

Etherington J.R. y McDonald I.R. Is Bitumen a Petroleum Reserve? SPE Annual Technical Conference and Exhibition, 26-29 de septiembre, 2004, Houston, Texas.

Etherington J.R. Getting Full Value from Reserves Audits, SPE Annual Technical Conference and Exhibition, 9-12 de octubre, 2005, Dallas, Texas.

Etherington J.R. Managing Your Business Using Integrated PRMS and SEC Standards, SPE Annual Technical Conference and Exhibition, 4-7 de octubre, 2009, New Orleans, Louisiana.

Farzin Y.H The Impact of Oil Price on Additions to US Proven Reserves. Resource and Energy Economics, volumen 23 (número 3), julio de 2001: 271-292.

Harrell D.R. Achieving Global Acceptance of and Compliance With a Universal Set of Petroleum Resources and Reserves Definitions: Are We There Yet? SPE Unconventional Reservoirs Conference, 10-12 de febrero, 2008, Keystone, Colorado, USA.

Hodgin J. y Harrell R. Assessment of World Petroleum Supply Enhanced by New Industry Resources Definitions, Offshore Technology Conference, 30 de abril al 3 de mayo, 2007, Houston, Texas.

Lajous A. Las reservas de hidrocarburos de Chicontepec, Nexos, México, septiembre, 2011.

Lee W.J. Modernization of the SEC Oil and Gas Reserves Reporting Requirements, SPE Annual Technical Conference and Exhibition, 4-7 octubre, 2009, New Orleans, Louisiana, 2009a.

Lee W.J. Reserves in Nontraditional Reservoirs: How Can We Account for Them? Journal SPE Economics \& Management, volumen 1 (número 1), octubre de 2009: 11-18, 2009b.

Lee W.J. The Reliable Technologies Rule: What Did the SEC Intend

Offshore Technology Conference, 3-6 de mayo, 2010, Houston, Texas, USA.

Maugeri L. Oil: Never Cry Wolf--Why the Petroleum Age Is Far from Over. Science, volumen 304 (número 5674), 21 mayo de 2004: 1114-1115.

Olsen G.T., Lee J.W. y. Blasingame T.A. Reserves Overbooking: The Problem We're Finally Going to Talk About, SPE Annual Technical Conference and Exhibition, 19-22 de septiembre, 2010, Florencia, Italia.

Patricelli J.A., y McMichael C.L. An Integrated Deterministic/Probabilistic Approach to Reserve Estimations. Journal of Petroleum Technology, volumen 47 (número 1), 1995: 49-53.
Pemex. Reservas de hidrocarburos en México 2010, México DF [en línea]. Disponible en: http://www.ri.pemex.com/files/content/ Libro\%202010.pdf

Pemex. Reservas de hidrocarburos en México 2011, México DF [en línea]. Disponible en: http://www.pemex.com/informes/pdfs/ reservas_hidrocarburos_2011.pdf

Salameh Mamdouh G. How Realistic are Opec's Proven Oil Reserves? Petroleum Review, volumen 58 (número 691), 2004: 26-29.

Securities and Exchange Commission. Modernization of Oil and Gas Reporting (17 CFR Parts 210, 211, 229, and 249; [Release Nos. 33-8995; 34-59192; FR-78; File No. S7-15-08], RIN 3235AK00, enero 1o, 2010 [en línea]. Disponible en: http://www. sec.gov/rules/final/2009/33-8995fr.pdf

Sener. Disposiciones administrativas de carácter general en materia del proceso para la publicación y registro de las reservas de hidrocarburos del país, Diario Oficial de la Federación, viernes 19 de noviembre, 2010 [en línea]. Disponible en: http://www.dof.gob.mx/nota_detalle.php?codigo=5167865\&f echa $=19 / 11 / 2010$

Society of Petroleum Engineers, American Association of Petroleum Geologists, World Petroleum Council, Society of Petroleum Evaluation Engineers, 2009. Petroleum Resources Management System, United States of America, 2007, 49p. [en línea]. Disponible en: http://www.spe.org/industry/docs/Petroleum_Resources_Management_System_2007. pdf\#redirected_from=/industry/reserves/prms.php

Society of Petroleum Engineers, American Association of Petroleum Geologists, World Petroleum Council, Society of Petroleum Evaluation Engineers and Society of Exploration Geophysicists, 2011, Guidelines for Application of the Petroleum Resources Management System, USA, noviembre, 221p. [en línea]. Disponible en: http://www.spe.org/industry/docs/PRMS_Guidelines_Nov2011.pdf

Virine L. y MacDonald D. Methodology for Integrated Reserves Management: Providing Regulatory Compliance and Improving Decision Making, SPE Annual Technical Conference and Exhibition, 4-7 de octubre, 2009, New Orleans, Louisiana.

Worthington P.F. Reserves-Getting It Right, International Petroleum Technology Conference, 21-23 de noviembre, 2005, Doha, Qatar.

\section{Bibliografía}

Comisión Nacional de Hidrocarburos. Nota adjunta a resolución CNH.E.03.001/11, 27 de mayo de 2011b [en línea]. Disponible en: http://www.cnh.gob.mx/_docs/resoluciones/Nota_Adjunta_a_Resolucionv2.pdf

Comisión Nacional de Hidrocarburos. Nota complementaria a resolución CNH.E.03.001/11, 31 de mayo de 2011c [en línea]. Disponible en: http://www.cnh.gob.mx/_docs/resoluciones/ Nota_complementaria_VFinal.pdf 
Etherington J.R. Can You Have Probable Without Proved Reserves? SPE Annual Technical Conference and Exhibition, 26-29 de septiembre, 2004, Houston, Texas

Haider-Ghazi M. World Oil Reserves: Problems in Definition and Estimation. OPEC Review, volumen 24 (número 4), 2000: 305327.

Harrell D.R. Current Applications of the Standards Pertaining to the Estimating and Auditing of Oil and Gas Reserves Information to Meet the Objectives of the Sarbanes-Oxley Act of 2002, SPE Annual Technical Conference and Exhibition, 5-8 de octubre, 2003, Denver, Colorado.

Society of Petroleum Engineers and World Petroleum Council. Petroleum Reserves Definitions, 1997, United States, 4p [en línea]. Disponible en: http://www.spe.org/industry/docs/Petroleum_Reserves_Definitions_1997.pdf

Society of Petroleum Engineers, American Association of Petroleum Geologists and World Petroleum Council. Petroleum Resources Classification System and Definitions, United States, febrero, 2000, 4p [en línea]. Disponible en: http://www. spe.org/industry/docs/Petroleum_Resources_Classification_2000.pdf

Society of Petroleum Engineers, American Association of Petroleum Geologists and World Petroleum Council. Glossary of
Terms Used in Petroleum Reserves/Resources Definitions, United States, 2004, 14p. [en línea]. Disponible en: http://www.spe.org/industry/docs/GlossaryPetroleumReserves-ResourcesDefinitions_2005.pdf

Yerguin D. In Search of Reasonable Certainty: Oil and Gas Reserves Disclosure, Cambridge Energy Research Associates. Oil \& Gas Journal, 25 febrero de 2005.

\section{Este artículo se cita:}

\section{Citación estilo Chicago}

Rodríguez-Padilla, Víctor. Sistema de estimación, certificación y aprobación de reservas de hidrocarburos en México; análisis de desempeño. Ingeniería Investigación y Tecnología, XIV, 03 (2013): 451-460.

\section{Citación estilo ISO 690}

Rodríguez-Padilla V. Sistema de estimación, certificación y aprobación de reservas de hidrocarburos en México; análisis de desempeño. Ingeniería Investigación y Tecnología, volumen XIV (número 3), julio-septiembre 2013: 451-460.

\section{Semblanza del autor}

Víctor Rodríguez-Padilla. Es físico y maestro en ingeniería energética por la Universidad Nacional Autónoma de México. Tiene el grado de doctor en economía de la energía por la Universidad de Grenoble, Francia. Obtuvo posgrados en el Institute d'Économie et Politique de l'Énergie (IEPE), Francia y en el Institute National de la Recherche Scientifique (INRS-Énergie), Québec, Canadá. Es especialista en contratos petroleros, régimen fiscal, economía de los hidrocarburos y política energética. 